\title{
CORRIGENDUM
}

\section{Rituximab in combination with high-dose methylprednisolone for the treatment of chronic lymphocytic leukemia}

JE Castro, DF James, JD Sandoval-Sus, S Jain, J Bole, L Rassenti and TJ Kipps

Leukemia (2009) 23, 2326; doi:10.1038/leu.2009.232

Correction to: Leukemia (2009) 23, 1779-1789;

doi:10.1038/LEU.2009.133

Since the publication of this paper, the authors have noticed an error.

On page 4 of the article, under the heading 'Hematologic and immune parameters', the last sentence should read:
'Moreover, the patients' median posttreatment serum IgA level of $32 \mathrm{mg} / 100 \mathrm{ml}( \pm 26$, s.d.) was significantly less than the median pretreatment serum IgA level of $47 \mathrm{mg} / 100 \mathrm{ml}( \pm 44$, s.d.; $P<0.05$, Student's $t$-test).'

The authors would like to apologize for any inconvenience caused. 